\title{
Determinación del Relieve 3D de la Superficie de Marte usando un UAV
}

\section{Mars Surface 3D relief using a UAV}

\author{
Jimmy Gora \\ Universidad Tecnológica del Perú, Lima 001
}

DOI: https://doi.org/10.33017/RevECIPeru2015.0005/

\section{Resumen}

En este artículo se presenta el desarrollo de un UAV (Quadcopter) para la determinación del relieve en 3D de la superficie del planeta Marte, el propósito de esta investigación es proveer una herramienta moderna y confiable a los tripulantes en futuras exploraciones espaciales, los cuales tendrán la oportunidad de enviar al equipo para realizar el reconocimiento y mapeo de un área específica. El objetivo del desarrollo de este dispositivo es asegurar la integridad física de las personas en cualquier misión de investigación y exploración de lugares desconocidos, ya que podrá ser monitoreado remotamente y brindar información relevante previa a la exploración humana lo que dará una mejor visión del terreno a investigar. Este proyecto fue desarrollado en el programa de experimentación y simulación en el MDRS (Mars Desert Research Station) organizado por The Mars Society, estación ubicada en el desierto de Utah, Estados Unidos, en este lugar se desarrolló también la simulación de supervivencia marciana. El desarrollo del equipo constó de dos etapas principales, la primera parte se enfocó en el análisis de hardware y software para el Quadcopter, se seleccionaron las diferentes piezas, el frame principal y los parámetros de control para un vuelo estable, la segunda parte se orientó a la creación del algoritmo para el procesamiento de las imágenes y la determinación del relieve en 3D, todo ello desarrollado en Matlab. En la estación de investigación MDRS se desarrollaron las simulaciones, pruebas de vuelo y toma de datos. El Quadcopter puede ser piloteado usando un control remoto para mapear diversas zonas, además de ello, gracias al GPS con el que cuenta, tiene la capacidad de establecer puntos en el aire para realizar un recorrido predeterminado y mapear una región definida. El principio que se emplea en el procesamiento de imágenes es la fotogrametría, la cual emplea fotografías digitales, mediante las cuales se realiza un reconocimiento de patrones y puntos de referencia para poder determinar la superficie fotografiada en 3D. Una cámara GoPro fue utilizada para la recolección de imágenes ya que brinda fotografías de buena calidad además de ser resistente a ambientes adversos como el polvo y a humedad. Las zonas contiguas a la estación en el Desierto de Utah fueron los lugares de experimentación, lo cual sirvió para poder calibrar los parámetros de control de vuelo del Quadcopter y los del algoritmo para el procesamiento de imágenes en Matlab. Los resultados obtenidos durante las primeras pruebas fueron óptimos, lo que demuestra que los métodos utilizados son los adecuados, pero aún no se han alcanzado resultados precisos, ya que se tiene un porcentaje de error mayor al esperado. Actualmente se viene trabajando en el mejoramiento del hardware del Quadcopter y en el programa de procesamiento de imágenes para obtener mejores resultados. Este trabajo es un aporte a la literatura científica en el área de exploración espacial, ya que este tema no ha sido muy desarrollado, se espera que esta investigación sirva como base para el desarrollo de nuevos aparatos relacionados a este contenido.

Palabras clave: Drone, UAV, Marte, relieve 3D, procesamiento de imagen, fotogrametría.

\section{Abstract}

In this article its presented the development of an UAV (Quadcopter) about 3D relief obtainment of Mars surface, the purpose of the research it to provide a modern and reliable tool to crewmembers on future space explorations, which will have the opportunity of send this machine to perform recognizing and mapping mission 
of an specific area. The main objective about the development of this device is to ensure the phisic integrity of people in any research mission or exploration mission of unknown places. It could be driven remotely and receive precise relevant information previously to the human exploration, which give a better vision of the researched area. This project was developed in the simulation program at MDRS (Mars Desert Research Station) organized by The Mars Society, the station is located in Utah desert in the USA. In that place was performed the simulation of mars survival. The development of the entire project consisted into two main stages, the first part was focused on hardware and software analysis of the Quadcopter. It was selected the main components, main frame and control parameters for a stable flight. The second part was oriented to the creation of an image processing algorithm and 3D relief obtaining, which was built on Matlab. On the Research Station the simulations, flight proofs and data collection were performed. The Quadcopter could be operated using a remote controller for mapping many zones, also it is provided of a GPS unit, which allow to set points in the air and follow a given range recording a defined region. The principle used in image processing is called photogrammetry, which use digital photos. Then, patterns and reference points are recognized to define the photographed surface into a 3D model. A GoPro camera was used to get pictures because this kind of devices are very resistant to dust and humidity also it gives high quality photos, important for the subsequent analysis. Close places to the station in the Utah desert were experimentation places. It helped to calibrate control flight parameters of quadcopter and image processing parameters on Matlab. Reached results during first tests were optimum, which demonstrate that methods used are correct, but precise results haven't reached yet, because there is an average error percentage. Currently it's been working on the improvement of the Quadcopter hardware and in the image processing program to get better results. This work is a contribution to the scientific literature in the space exploration field. This specific topic was not well developed, so it's hoped that it serve as base for many new projects and the development of innovative devices related to this.

Keywords: Drone, UAV, Mars, 3D relief, image processing, photogrammetry.

\section{Introducción}

La exploración espacial es una de las grandes disciplinas que actualmente está en un prominente desarrollo, la búsqueda de nuevos horizontes en el universo hacen que cada vez se desarrollen nuevos aparatos tecnológicos que nos brinden más y mejor información, la motivación básica para realizar esta investigación es la curiosidad y el deseo de conocer más de cerca el entorno del planeta Marte y del misterioso universo en general.

Actualmente los UAV o comúnmente llamados drones están siendo utilizados en diferentes áreas debido a su versatilidad y bajo coste, es por ello que en este trabajo se emplea un Quadcopter para sobrevolar y obtener información fotográfica de la superficie. El desarrollo de este quipo tiene como propósito brindar una novedosa herramienta para la futura tripulación que viaje al planeta rojo, en el cual sirva de apoyo a las exploraciones realizando el reconocimiento de terrenos, previos a su exploración humana.

La falta de equipo especializado para un mapeo con alta precisión de áreas geográficas es el tema principal a desarrollar. La información brindada actualmente por los satélites y rovers de Marte, no cuenta con la exactitud necesaria para la reconstrucción del relieve en 3D a escala.
Al ser uno de los objetivos de los nuevos equipos de exploración, asegurar la integridad física de las personas en cualquier misión de investigación de lugares desconocidos, la relevancia que tiene el equipo es alta.

Los datos a ser analizados serán las fotografías tomadas por la cámara, la cual está montada debajo del Quadcopter. La fotogrametría aérea es el principal método a emplear, la cual consiste en el análisis de las fotografías, determinando puntos específicos los cuales sirven como referencia para el procesamiento de imágenes y la obtención del relieve 3D.

Este trabajo contribuye a la literatura científica del área de exploración espacial enfocada en el planeta Marte. Con el desarrollo y la experimentación progresiva del equipo se espera obtener el relieve en 3D de la superficie de Marte.

La experimentación y la obtención de superficies se realizaron en zonas contiguas a la Estación de Simulación del Desierto de Marte (MDRS) de The Mars Society ubicado en el desierto de Utah, EEUU, lugar en el cual se desarrolló también la simulación de supervivencia marciana. 




Fig. 1 MDRS, Utah, EEUU.

Los resultados obtenidos indican que si bien los métodos empleados son correctos, la no complejidad del análisis hace que el producto no tenga la calidad esperada.

En la sección 2 se describen los conceptos de los temas principales a desarrollarse en este artículo, en la sección 3 se detallan los métodos empleados para la construcción del Quadcopter y la determinación del relieve 3D en base al análisis de imágenes, en la sección 4 se presentan los resultados, seguidamente se presentan la discusión y las conclusiones en las secciones 5 y 6 respectivamente.

\section{Marco Teórico}

\subsection{Quadcopter}

\subsubsection{UAV}

Unmanned Aerial Vehicle, es un equipo no tripulado con la capacidad de realizar distintas actividades, desde propósitos militares hasta ayuda humanitaria, pudiendo ser controlado remotamente o de manera autónoma.

\subsubsection{Quadcopter}

Es un tipo de UAV, el cual consta de 4 rotores con sus respectivas hélices. La variación de la revoluciones RPM de cada rotor determina el movimiento del vehículo.

\subsubsection{APM}

Ardupilot Mega, es el controlador utilizado en el equipo. Este controlador está basado en Arduino, el cual nos brinda muchas ventajas tales como programar vuelos según una trayectoria, ajustar parámetros de control y la calibración general de todas las partes del UAV.

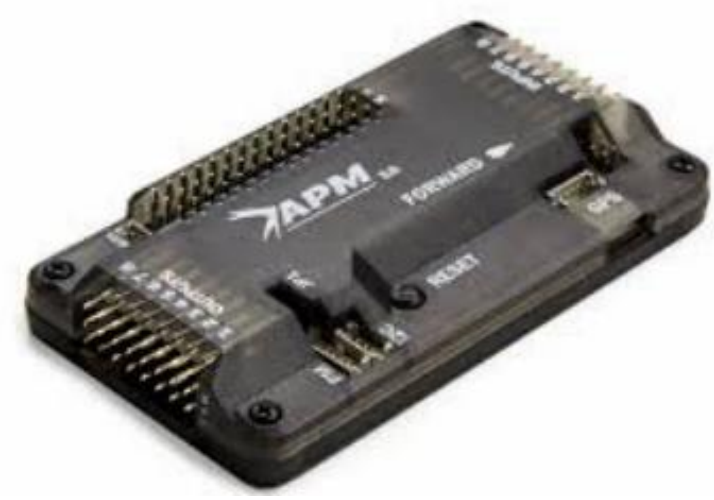

Fig. 2 Ardupilot Mega 2.6.

\subsubsection{UAV Frame}

Es el esqueleto del Quadcopter, sobre el cual se montan las demás piezas tales como, los motores brushless, el controlador, los variadores de velocidad, la batería, la cámara, los sensores, etc.

\subsubsection{Cámara}

Para la adquisición de fotografías del área a analizar se optó por una GoPro de 12 megapíxeles, una resolución óptima para el análisis.

\subsubsection{Mission Planner}

Se describe el procedimiento experimental y el equipamiento utilizado. Aquí no se muestran los resultados sino se describe la metodología empleada en el trabajo experimental.

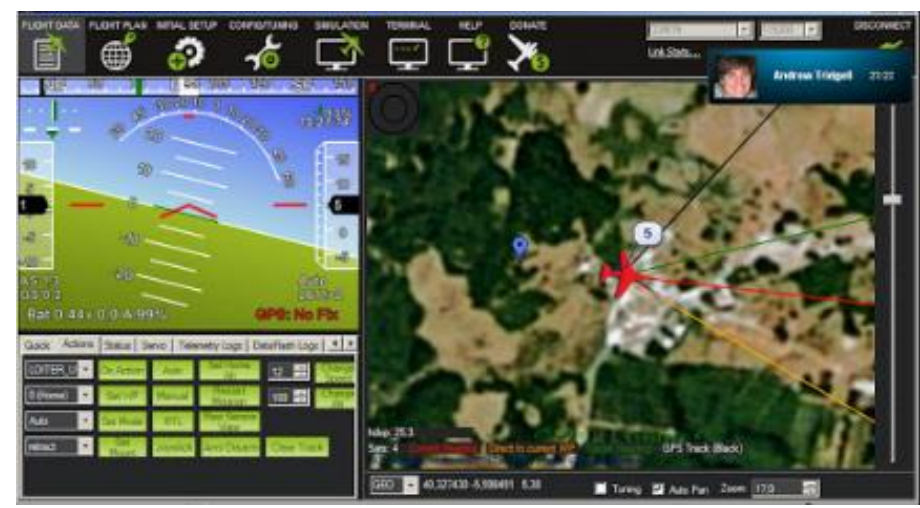

Fig. 3 Mission Planner 1.3.19.

\subsection{Fotogrametría}

La fotogrametría aérea utiliza fotos aéreas tomadas desde una plataforma especial (UAV). 


\subsubsection{Fotogrametría}

Es el método empleado para determinar modelos en $3 \mathrm{D}$, es decir información medible de imágenes reales [1] a partir de fotografías usando relaciones, matemáticas de geometría proyectiva y visión estereoscópica.

\subsubsection{Visión Estereoscópica}

Este tipo de visión permite obtener impresión de profundidad al observar dos imágenes enfocadas desde distintos ángulos de un mismo objeto.

\subsubsection{Orientación Interna}

Es el proceso por el cual se reconstruyen los haces de los rayos que originaron la fotografía.

\subsubsection{Orientación Externa}

Proceso por el cual se genera el modelo estereoscópico, mediante la intersección de los pares de rayos homólogos, se obtiene el modelo tridimensional dándole a la una escala y la nivelación respectiva.

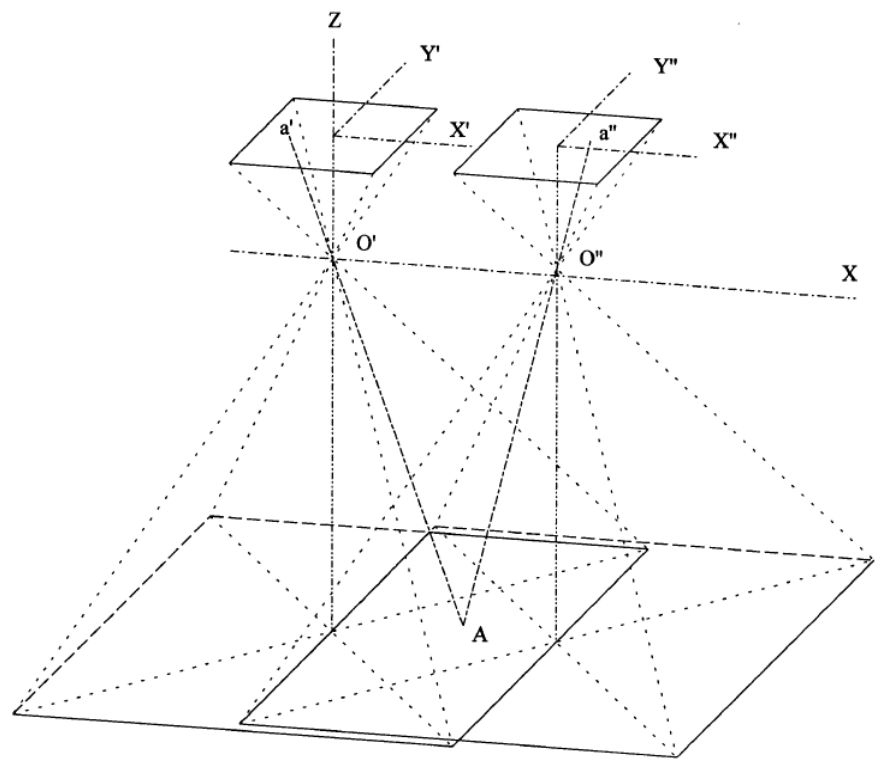

Fig. 4 Visión estereoscópica desde los puntos $Y^{\prime} y$ $Y^{\prime \prime}[2]$.

\section{Métodos}

\subsection{Ensamble del Quadcopter}

Un punto importante para el desarrollo de la investigación es el Quadcopter, ya que este será el encargado de servir como plataforma a la cámara que recabará información de la superficie a analizar [3].

El equipo está compuesto por un Frame o armazón sobre el cual se van a montar todos los elementos necesarios para el vuelo. Se emplearon 4 motores Brushless trifásicos en conjunto con 4 hélices para la propulsión de la nave. Dado que los motores son trifásicos, su modo de control no es tan sencillo como el de un motor DC simple, es por ello que se requieren variadores de velocidad (ESC), los cuales reciben la señal de control y la transforman a una señal compatible con los motores.

El controlador empleado es un Ardupilot Mega 2.6, debido a su versatilidad, rápido crecimiento y extensión de esta plataforma [4], es uno de los controladores más empleados para vehículos de exploración UAVs, Rovers, etc. Asociado a un giroscopio y un GPS el controlador nos brinda la información necesaria para poder determinar la posición de las fotografías tomadas y la ruta de vuelo del Quadcopter. El fácil ajuste de parámetros es uno de los puntos más importantes de este controlador ya que nos permite calibrar el control y por ende la estabilidad para que la toma de datos sea la más precisa posible.

Todo el equipo a excepción de la cámara esta alimentado por una batería de Polímeros de Litio LiPo, debido a la estabilidad de energía y la capacidad de almacenamiento de este tipo de baterías son las más usadas en UAVs. Las figuras 5 y 6 muestran las partes del Frame del Quadcopter y su montaje final respectivamente.



Fig. 5 Partes del Frame del Quadcopter, previo al ensamble. 


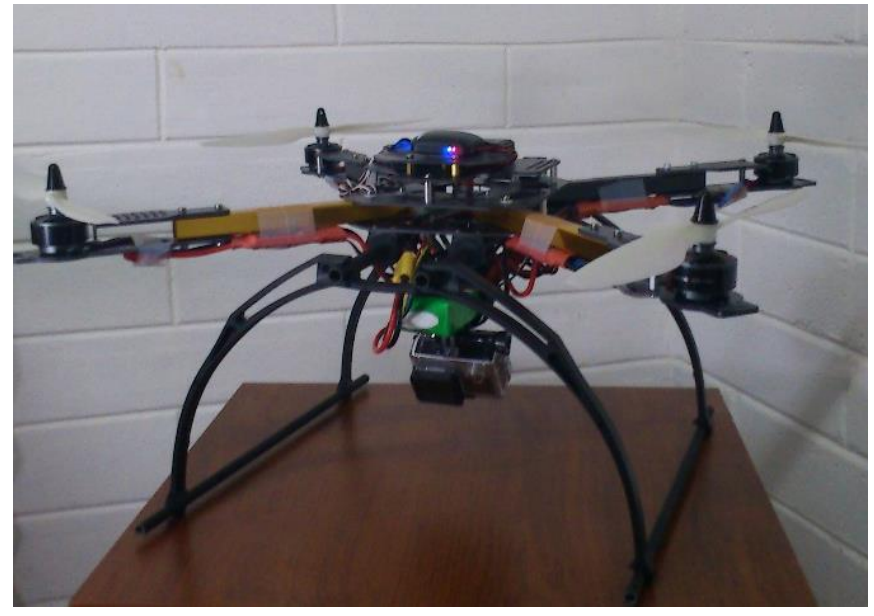

Fig. 6 Ensamble final del Quadcopter.

\subsection{Calibración del Quadcopter}

Es fundamental la buena calibración y ajuste de parámetros del equipo para así poder tener un sistema estable y confiable en la adquisición de datos. Para la calibración del Quadcopter se va a ingresar al programa Mission Planner, una vez dentro se procederá a la carga del firmware correspondiente dependiendo de la disposición de los rotores y el modelo de vehículo a emplear, en la figura 7 se muestra la pantalla inicial para seleccionar el modelo.

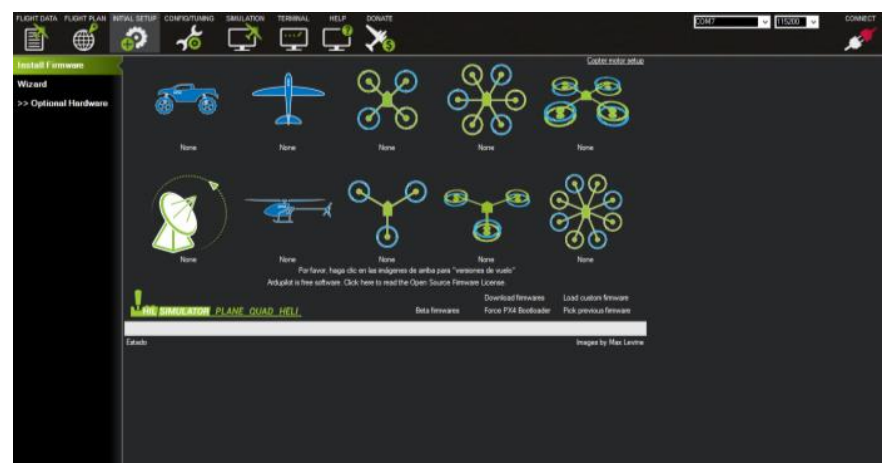

Fig. 7 Selección de modelo en el software Mission Planner.

Se ajustaran además las condiciones de vuelo, la altitud de vuelo y de ser necesario un recorrido predeterminado para sobrevolar una zona determinada.

\subsection{Adquisición de Datos}

La adquisición de datos será realizada a través de la cámara fotográfica montada en el Quadcopter. La GoPro es una de las cámaras más empleadas en deportes de aventura e investigación debido a su robustez ante ambientes adversos. Además de ofrecer una gran calidad de fotos aéreas y videos en alta resolución, sus reducidas dimensiones permiten su fácil montaje en diversos equipos e incluso sobre el cuerpo humano.

El la figura 8 se puede apreciar la cámara GoPro con su respectiva protección para resistir condiciones ambientales adversas.

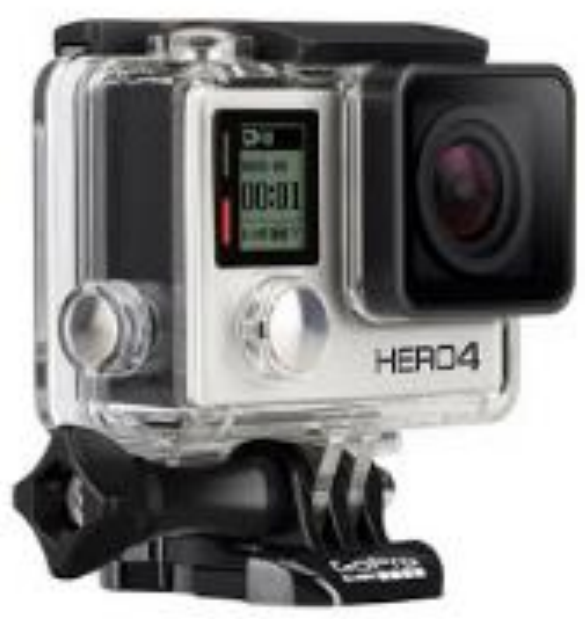

Fig. 8 GoPro Hero 4 Silver.

La toma de fotografías se realizó, capturando dos fotografías desde cada punto elegido, de tal manera que se tengan dos opciones para poder elegir la mejor y más nítida.

La cámara tiene una resolución de 12 Megapíxeles lo cual nos da una dimensión de 4000 × 3000 por cada fotografía.

La determinación de la altura de toma de fotos se estableció ajustando los parámetros en el controlador de vuelo del Ardupilot.

\subsection{Procesamiento Digital de Imágenes}

El procesamiento de las imágenes captadas por la GoPro se realiza en el programa Matlab, el cual nos provee de un entorno para el análisis de las imágenes [5] tomadas por el Quadcopter.

Mediante la creación de algoritmos que permitan generar el relieve en $3 \mathrm{D}$, el procesamiento de los datos se realizó de la siguiente manera:

Se analizó el brillo y contraste de cada fotografía para que el procesamiento sea lo más parejo posible y tener la mayor cantidad de datos válidos., para ello se ajustaron los histogramas de cada imagen. 
Luego se realizó la detección de puntos en el área común entre dos fotografías contiguas para poder determinar su ubicación exacta, para ello se hizo uso de desplazamientos y giros variando así su orientación y determinando las alturas relativas a cada pixel de la imagen.

Finalmente se usa la correlación de imágenes para determinar todos los puntos coincidentes en las imágenes y generar el relieve en 3D.

Luego de haber obtenido los relieves en 3D, se procedió a realizar un análisis de precisión, para lo cual se tomaron las medidas reales entre dos puntos conocidos en la superficie del terreno, de la misma manera mediante un algoritmo se determinó la distancia entre los mismos puntos en el relieve 3D obtenido. Este análisis se realizó para tres altitudes diferentes de toma de datos; 7, 10 y 12 metros sobre el nivel promedio de la superficie.

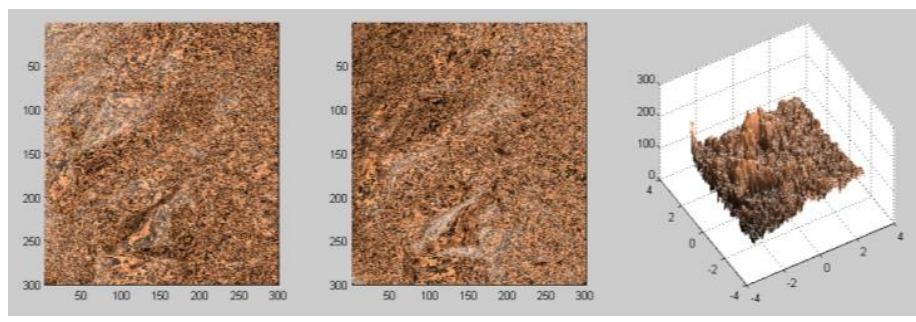

Fig. 9 Dos imágenes continuas y la reconstrucción en $3 D$.

\section{Resultados}

Se presentan los resultados del procesamiento de imágenes, la figura 10 muestra el relieve 3D obtenido de la superficie del desierto de Utah, lugar en el cual se llevaron a cabo las pruebas.



Fig. 10 Resultado del análisis de imagen.
Además de ello se realizó un análisis de relievealtitud, en el cual se analiza la precisión del Relieve vs. La altitud de toma de fotografías. La Tabla 1 muestra los diferentes porcentajes de precisión a diferentes alturas.

\begin{tabular}{|c|c|c|c|}
\hline Altitud (m) & 7.0 & 10.0 & 12.0 \\
\hline Precisión (\%) & 63.7 & 52.9 & 41.2 \\
\hline
\end{tabular}

Tabla 1 Precisión de relieve en base a la altitud de toma de fotografías.

Cabe mencionar que a medida que la toma de fotografías se realizaba a mayor altitud se observó que la inestabilidad del Quadcopter aumentaba.

\section{Discusión}

Los resultados obtenidos fueron los esperados, pero se dedujeron 3 factores principales que no contribuyeron en un buen sistema:

El primero es el ajuste de parámetros de control, el cual deberá ser mejorado con un tipo específico de control para obtener mayor estabilidad en el vuelo.

- $\quad$ El segundo es que el Quadcopter actual no posee una superficie aerodinámica que ayude a la estabilidad teniendo un mejor flujo de aire sobre y debajo el recubrimiento.

Finamente mejorar la generación del relieve en 3D mediante el empleo de técnicas más robustas de procesamiento de imágenes.

\section{Conclusiones}

Del presente trabajo de investigación se concluye que se determinó el relieve en 3D de las superficies analizadas, teniendo así una nueva herramienta en exploración aeroespacial.

Se tiene como futuros objetivos de la segunda parte de este trabajo de investigación la determinación de una superficie aerodinámica, el ajuste de parámetros de control para estabilidad de vuelo y la optimización del procesamiento de imágenes para una mayor precisión.

Luego de determinar los puntos anteriores, se van a realizar análisis en condiciones similares a Marte en cuanto a presión, gravedad y factores ambientales. 


\section{Agradecimiento}

Se brinda un agradecimiento especial a The Mars Society por haber admitido al autor de este artículo a formar parte del Crew 150 "Team Peru III", realizando una Simulación de Supervivencia en Marte en el Mars Desert Research Station - MDRS del desierto de Utah, EEUU entre los meses de Febrero y Marzo de 2015, donde se presentó este proyecto y se realizaron las pruebas del equipo.

\section{Referencias}

[1] Saxena A. y Sun M., "Learning 3D Scene Structure from a Single Still Image". IEEE Transactions on Pattern Analysis and Machine Intelligence, 2009, 31(5): 824-840.

[2] J. Santamaría y T: Sanz, "Fundamentos de Fotogrametría", Servicio de Publicidad
UNIRIOJA, Universidad de la Rioja, La Rioja, España 2011.

[3] K. Afrendy, A. Ahmad, A. Manan, K: Nizam y W: Sofia, "Integration of low Altitude Aerial \& Terrestrial Photogrammetry Data in 3D Heritage Building Modeling", in IEEE Control and System Graduate Research Colloquium, 2012, paper 5, p. 34.

[4] J. Dandois y E. Ellis, "High Spatial Resolution Three-Dimensional Mapping of Vegetation Spectral Dynamics using Computer Vision", Remote Sensing of Environment, vol. 136, pp. 259-276, 2013.

[5] S. Madeira, J. Concalves y L: Bastos, "Photogrammetric Mapping and Measurement Application using MATLAB", Computers and Geosciences, vol. 36, pp. 699-706, 2010.

E-mail: jimmy.r.gora@gmail.com 